\section{Preparing Micrographs and Applying Scale Bars Using Adobe Photoshop Elements $^{\mathrm{TM}}$}

\author{
Susan A. Lancelle \\ Mount Holyoke College, South Hadley, MA \\ slancell@mtholyoke.edu
}

As part of our introductory Cell Biology course, our students acquire images through microscopes using consumer cameras with memory cards. They then transfer the images to a computer and prepare them using Adobe Photoshop Elements. ${ }^{\mathrm{TM}}$ An important part of this process is learning to apply a scale bar, which calibrates the specimen image. Photoshop Elements is a relatively cheap and widely available software package that easily accomplishes this task for anyone who is using a camera system or software that does not automatically apply a scale bar or calibration data to the image. The instructions and figures herein refer to Photoshop Elements version 4.0 , but they apply to other versions of the software.

Overview of the method: Students either make images of a stage micrometer with each appropriate objective at their workstation, or have these images available to them in a folder on the computer. Each workstation should have its own calibration images. These stage micrometer calibration images should be identified by suitable name (Workstation_1_40x, for example) for the camera, microscope,

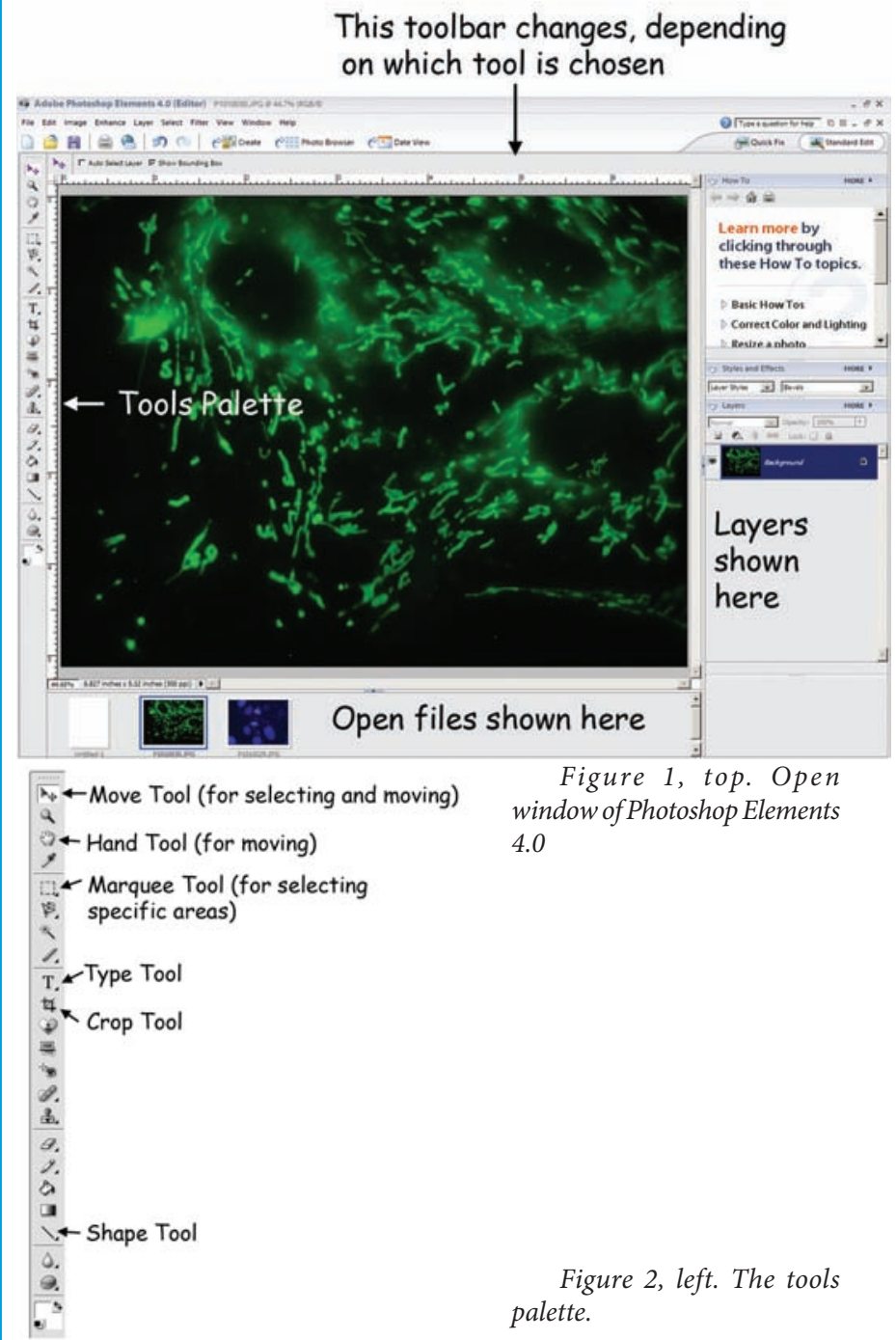

and objective, so that the correct image can be easily sel appropriate calibration image is "attached" to a specimen image by adding it as a "layer" so that it is non-destructively carried along with the specimen image as it is prepared for the final plate. Any resizing of the specimen image also affects the scale image layer. Finally the scale image serves as the basis for drawing a presentable scale bar on the specimen image layer during the annotation stage; the stage micrometer layer can then be removed.

Figure 1 shows an open window of Photoshop Elements, showing the basic layout. Figure 2 shows at higher magnification of the tools palette, with some of the more commonly used tools indicated.

1. Start Adobe Photoshop Elements 4.0. Open the image (File $>$ Open). Save this as the "original image" and from now on work on a copy. To do this, duplicate the image (File $>$ Duplicate) and give the new copy of the image a new name. Close the window with the original image; this way you will always have the original, unmodified image to return to if necessary. Notice that all open image files are shown in icon form at the bottom of the page; you can switch between them by clicking on the icons.

Throughout working with the images, remember that the Undo command (Edit $>$ Undo or use the Undo button) is your friend!

2. Be sure the rulers show on the image (View $>$ Ruler) and are in inches (on a PC, right click on ruler to change to inches; on a Mac, double click on the ruler to change to inches).

3. Now overlay an image of a stage micrometer taken with the same objective lens, microscope, and camera resolution that you used to take the picture you are currently editing. This must be done before you do any resizing of your image. Open the appropriate micrometer image (File>Open). You may wish to crop the image so that only a part of the micrometer is visible. To do this, click on the Crop Tool in the toolbox, click and drag the rectangle on the image to select the desired area to crop, then Image $>$ Crop. Now paste the cropped image into the original image: Select $>$ All, Edit $>$ Copy. Switch to the picture file; Edit>Paste.

Notes on working with Layers: Each element you add to the image, whether it is a new picture, text, or drawing, etc., is treated as a separate layer. These are visible on the right side of the window (if you don't see them, select Window>Layer and they will show). These layers are convenient in figure preparation because they allow you to manipulate one layer without disrupting the others. Click on a layer to activate and change it; right click on a layer and select "delete" to remove that layer (alternately, use the "layer" dropdown menu and select "delete"). Flattening the image will combine all of the layers into one, so don't do this until you are finished with all image manipulations and are certain you want to keep all changes. Layers cannot be changed or removed once the image is flattened.

Use the Move Tool to select and reposition the micrometer image by clicking and dragging it from the center point (Figure 3 ). Important: do not drag the handles of the micrometer image, or you will resize it and it will no longer be calibrated to your original specimen image (remember the Undo command!). You will eventually delete this micrometer image, but for now, keep it with your picture so if you resize the picture, the micrometer image will resize as well.

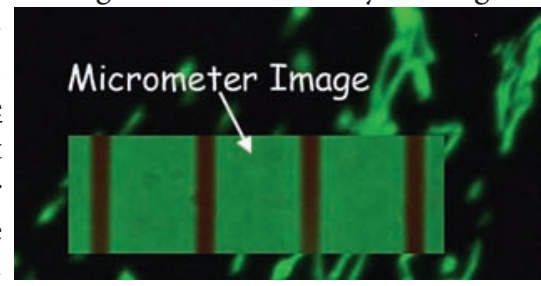

Figure 3. Stage micrometer image as a layer in the original image. 

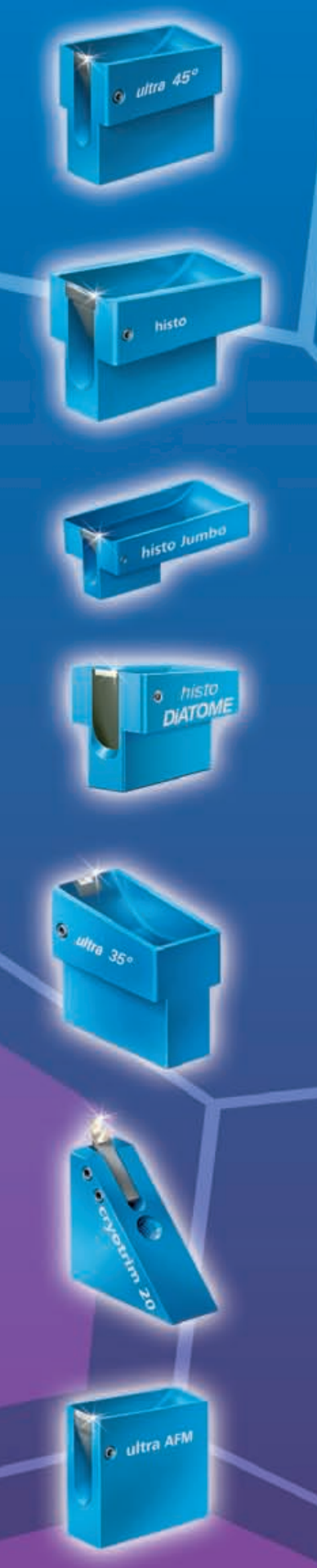

\section{cryotrim \\ 45 and 25 optimizing trimming with diamond blades.}

\section{ultra AFM \& cryo AFM}

the first diamond knives for AFM at room and low temperatures.

cryo $25^{\circ}$

for sectioning frozen hydrated specimens.

\section{STATIC LINE II}

the ionizer for eliminating electrostatic

charging in ultramicrotomy.

\section{DIATOME}

for all your sectioning requirements

\section{For more information, please call or white us today, or visit us online at:}

\section{What services can we offer you?}

Jechnical assistance in all fields of ultramicrotomy.

I Free sectioning tests for all types of samples.

Make use of our many years of experience in perfecting our knives.

Custom knives, tools, and boats.

I Special purchase programs.

Workshops and training.

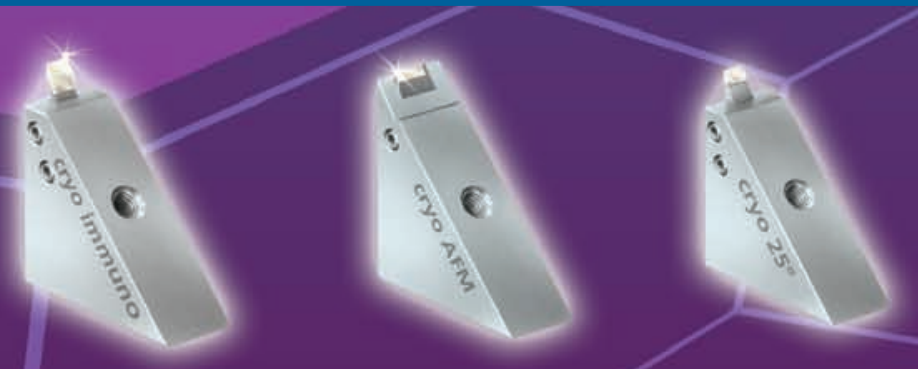

\section{ww.diatomeknives.com}

\section{P.O. Box 410 - 1560 Industry Rd.} Hatfield, Pa 19440

(215) 412-8390 • Toll Free: 1-(800) 523-5874

Fax: (215) 412-8450 or 8452 email:sgkcck@aol.com•stacie@ems-secure.com 
If desired, crop the image to eliminate unnecessary parts (see above). Do not accidentally crop out the image of the stage micrometer! Move it to an area of the picture you will keep before you crop the image.

4. Adjust the brightness and contrast if desired (Enhance $>$ Adjust Lighting $>$ Brightness/Contrast). Make sure preview box is checked and move the small triangles back and forth to try to improve the image. Clicking "Cancel" will return the picture to its original state.

5. In order to be consistent with the size of labels and type that you might apply to the image, reset the document size before you add these elements (Image $>$ Resize $>$ Image Size). Many cameras default to 72 dpi display resolution (typical for a computer monitor, but much too large for printing). First uncheck "Resample Image" and set the document resolution to 300 pixels/inch, which is near photographic quality. Check the final size of the image. If it is not the size you desire, check "Resample Image" and "Constrain Proportions" and type in the desired size of one of the dimensions of the figure; the other will automatically adjust. The scale micrometer will change along with the rest of the image. It is important to note that you want to avoid resampling the image whenever possible, as it changes the pixel dimensions of the picture, and can degrade the resulting image.

6. Add labels using the Type Tool in the tool bar. Click on the Type Tool and notice that various options appear at the top of the image. Use black or white font color and Arial font in an appropriate size, 12 or 14 pt. for a 300 dpi document size is suitable. Select the desired spot to label on the image, click and start typing. Notice the two icons that appear at the top right. The circle/slash is the "cancel" icon and the checkmark is the "commit" icon (see Figure 7). If you change your mind about where you want to place the type, click on the "cancel" icon; once you are satisfied with the label you have typed (its size, color, placement, etc.), you must click on the "commit" icon before you can move on. If you want to change the location of the text after you have committed, select it with the Move Tool and drag. Alternately, select the layer that contains that text, delete it (right click, delete) and start again.

7. Referring to Figures 2 and 4 , use the following procedure to add arrows to point out features in the image. Draw a line using the Line Tool in the tools palette. If the Line Tool isn't showing, click on the Shape Tool. A shape tool bar now becomes visible in the upper left of the screen; select the line. (Alternatively, you can right click on the Shape Tool and select the Line). To add arrowheads to the line, before you draw it, click the small arrow at the left side of the shape tool bar at the top of the screen to find a pull-down menu labeled "Geometry Options." Choose the arrowhead settings you would like to use. To change the thickness of the line, change the "Weight" of the line. Remember that to delete or change an item, you must select the layer it is in and then you can act on it.

8. Other changes you may want to make to a layer before the

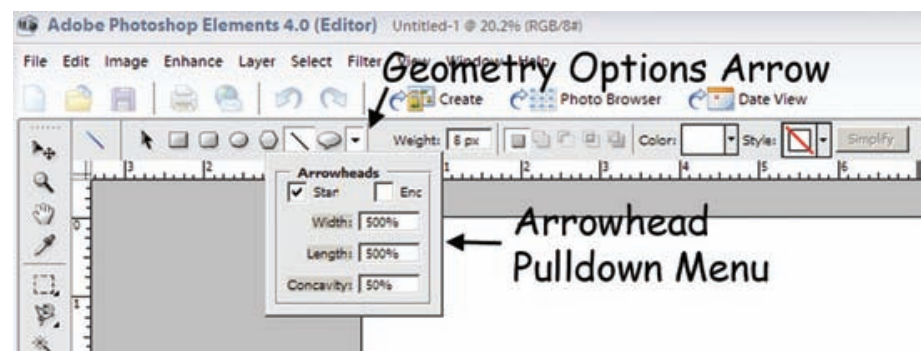

Figure 4. Tools for drawing arrows. image is flattened: To change size, font, color, etc. of text, select from the Layers Palette the layer that contains the text you want to change. Then use the text tool bar at the top of the screen to select the changes you want to make. Sometimes it is helpful to outline a letter or arrowhead with a contrasting color, e.g., outline a white object with black or vice versa. To do this, select the appropriate layer and simplify it (Layer $>$ Simplify). Then chose Edit $>$ Stroke (Outline) Selection. From the fly out menu that appears, choose the width and color of the outline, check "outside" and uncheck "preserve transparency." Experiment with your images, but save intermediate images along the way! Also, use the "Undo" command if you don't like the result!

Remember that if it seems Photoshop isn't letting you "move on," you may have to click on the "commit" or "cancel" icon first.

9. Now use the Line Tool (without an arrowhead!) to generate a scale bar. Refer to Step 7 for drawing lines. Here is where you use the image of the scale micrometer that you added earlier. Position it somewhere near the lower right corner of the image. Each division on a typical stage micrometer equals $10 \mu \mathrm{m}$. From the upper toolbar, select white or black for your line, depending on the darkness in that part of your image, and a thickness (weight) that will be easily visible. Click in the center of one of the scale markers and draw a line across one or more of the divisions, ending in the center of another marker. If you can't see the line you have drawn, it is because that layer is behind the layer containing the micrometer image. Select the layer containing the micrometer image, then Layer $>$ Arrange $>$ Send to Back. This should place that layer under all of the others, except the background layer. The number of divisions you cover will depend on the magnification of your image; don't make it so long that it is distracting. Using the Text tool, type a line of text just above the scale bar, indicating what its length represents (e.g., $10 \mu \mathrm{m}$ ). Figure 5 is a small corner of a much larger image, showing how to draw the line on the image of the stage micrometer.

10. Select the layer containing the original image of the stage micrometer and delete it by right-clicking on that layer and selecting "delete." As shown in Figure 6, the scale marker and text that you added remain, because they are in separate layers.

11. If you wish to insert your finished image into another file, as part of a set of figures for example, open a blank file (File > New) and set the resolution to be the same as your finished figure. To copy

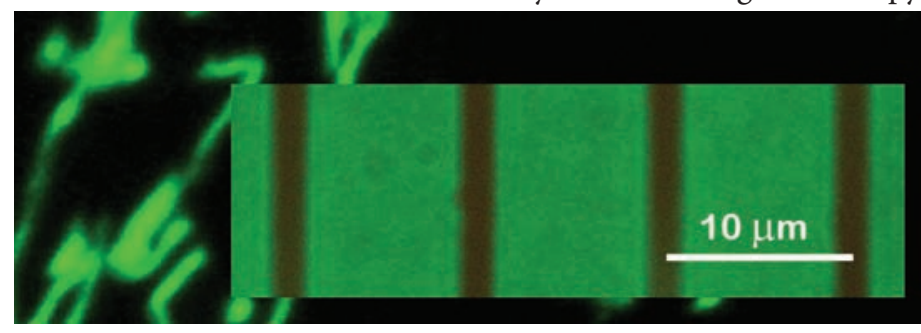

Figure 5. Scale bar drawn on the image of the stage micrometer.

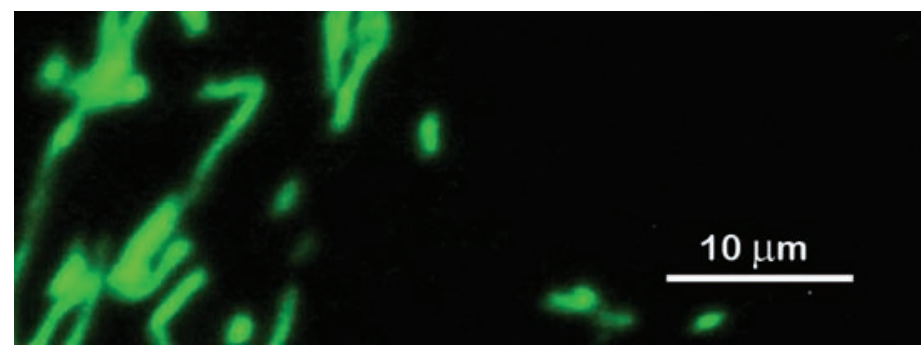

Figure 6. Completed scale bar with layer containing stage micrometer image removed. 


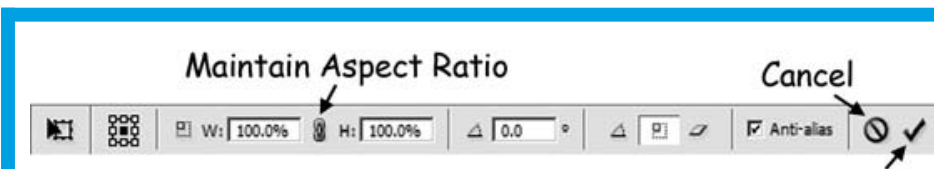

Commit

Figure 7. Scaling toolbar

and paste images into the blank document, after you have flattened the image, Select $>$ All, Edit $>$ Copy. Switch to the blank document, Edit $>$ Paste. Once you have pasted the image into the new document, do NOT change the size of the image by simply dragging the handles (the image will get distorted; if you accidentally do this, click the "cancel" icon and the image will return to its original size). If you want to change the size, follow the instructions for resampling in step 5 , or use Image $>$ Resize $>$ Scale. Then click on the "Maintain aspect ratio" icon (Figure 7 ). Now you can resize by clicking and dragging the handles, and the image will not be distorted. Alternatively, you can hold the shift key while clicking and dragging the handles, and the aspect ratio will be maintained. Click the "commit" icon once you are satisfied with the changes you made. Note, however, that once you commit, the image is resampled, so you don't want to resize more than necessary, or you risk significantly degrading your image.

The final image now contains a scale bar that has maintained the calibration of the original image through any manipulations performed along the way. While Photoshop Elements is not a scientific imaging program, we have found that it is convenient for student use because of the way it handles layers (each can be individually manipulated until the image is flattened). Using it to apply the scale bar eliminates the need to use more than one program to accomplish both labeling and calibration.
PELCO ${ }^{\circledR}$

\section{Silicon Nitride Membranes}

Next Generation

$\mathrm{Si}_{3} \mathrm{~N}_{4}$ TEM Support Films

- Durable and inert planar 50 and $200 \mathrm{~nm}$ substrates

- $3.0 \mathrm{~mm}$ circular frame

- EasyGrip ${ }^{\mathrm{TM}}$ edges

- Free from debris

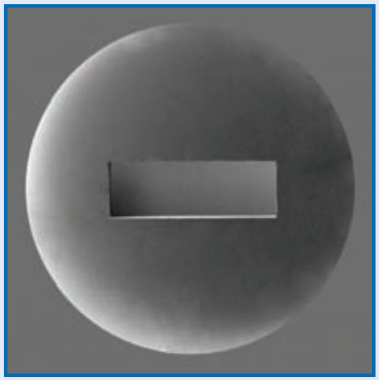

- Complimented with Holey Membranes and Silicon Dioxide Substrates

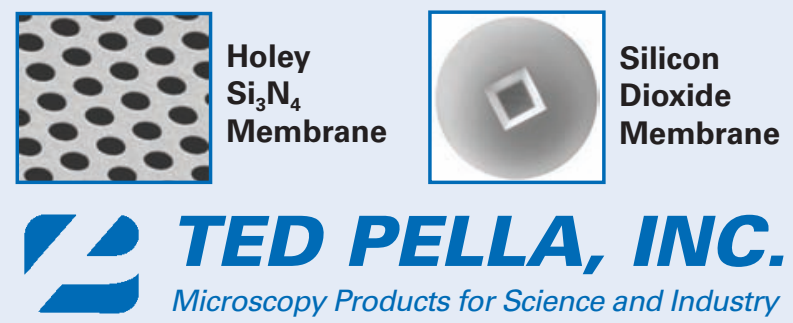

sales@tedpella.com 800-237-3526 www.tedpella.com

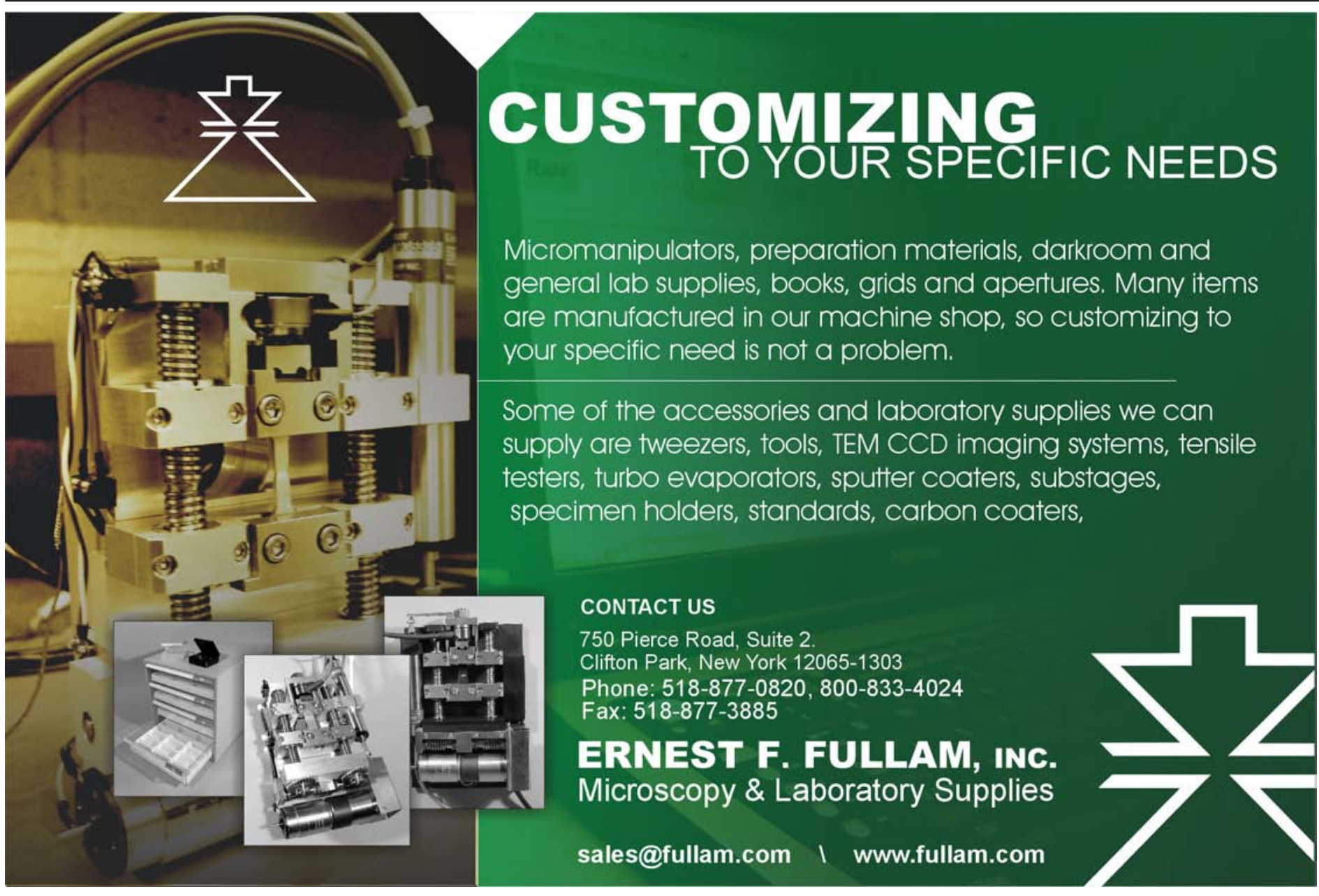

